\title{
Clinoposaponins I-V, New Oleanane-Triterpene Saponins from Clinopodium gracile O. KunTZE
}

\author{
Atsushi Yamamoto, Toshio Miyase, ${ }^{*}$ Akira Ueno and Toshio Maeda \\ School of Pharmaceutical Sciences, University of Shizuoka, 52-1, Yada, Shizuoka 422, Japan. \\ Received December 14, 1992
}

\begin{abstract}
From the whole plants of Clinopodium gracile five new saponins, called clinoposaponins $I-V$, were isolated together with two known and four artifact saponins, and their structures elucidated from spectroscopic data and chemical evidence. These saponins were composed of saikosaponin a and additional glucose moieties.
\end{abstract}

Keywords Clinopodium gracile; Labiatae; clinoposaponin; saikosaponin a; oleanane-triterpene saponin

In an earlier paper, ${ }^{1)}$ we reported the isolation and structure elucidation of saikosaponin a and buddlejasaponins I-IV, which were oleanane-triterpene saponins isolated from the methanol extract of Buddleja japoninca HEMSL (Buddlejaceae). These saponins were composed of saikosaponin a and one or two more sugar moieties. Until recently, saikosaponins had been isolated only from Bupleurum species (Umbelliferae). We thought that other species of plants might have saikosaponin-like saponins, and we obtained five new saponins, saikosaponin-like compounds, from Clinopodium gracile O. KunTZE (Labiatae). This paper describes the isolation and structure elucidation of these saponins.

A water extract of the whole plants of $C$. gracile was passed through a Mitsubishi Diaion HP-20 column, and absorbed materials were eluted with a $50 \%$ aqueous methanol mixture followed by methanol. The methanol eluate was rechromatographed on an octadecyl silica (ODS) column followed by repeated semi-preparative HPLC on an ODS column. We identified a total of 11 saponins.

Compounds 1 and 2 were identified by ${ }^{1} \mathrm{H}$ - and ${ }^{13} \mathrm{C}-\mathrm{NMR}$ spectra, FAB-MS, methanolysis and subsequent acid hydrolysis as saikosaponin a (1), ${ }^{2-4)}$ and buddlejasaponin IV (2), ${ }^{1)}$ respectively. Buddlejasaponin IV (2) was the main saponin of $C$. gracile and it accounted for $0.38 \%$ of the dried whole plants.

Clinoposaponin I (3) revealed an $[\mathrm{M}+\mathrm{Na}]^{+}$ion peak at $m / z 966$ in the FAB-MS and elemental analysis data was consistent with the molecular formula $\mathrm{C}_{48} \mathrm{H}_{78} \mathrm{O}_{18} \cdot 5 \mathrm{H}_{2} \mathrm{O}$. On mild methanolysis with $\mathrm{AcCl}-$ methanol $(1: 20), 3$ afforded saikogenin $\mathrm{A} .{ }^{3)}$ Subsequent acid hydrolysis of the methanolysate yielded fucose and glucose in the ratio of $1: 2$ as the sugar moiety. In the ${ }^{1} \mathrm{H}-\mathrm{NMR}$ spectrum, the proton signals of $\mathbf{3}$ were assigned by means of a detailed proton spin decoupling experiment (Table III). Based on this assignment, the protonated carbon signals were assigned by carbon-13-proton correlation spectroscopy $\left({ }^{13} \mathrm{C}-{ }^{1} \mathrm{H}\right.$ COSY) (Tables I and II). As far as the aglycone moiety was concerned, the ${ }^{1} \mathrm{H}-\mathrm{NMR}$ spectrum of $\mathbf{3}$ showed six singlet methyl proton signals $[\delta 0.91,0.92,0.94,1.00$, $1.11,1.39]$, two olefinic proton signals which were due to $\mathrm{H}-11[\delta 6.01(1 \mathrm{H}$, br d, $J=10 \mathrm{~Hz})]$ and $\mathrm{H}-12[\delta 5.66(1 \mathrm{H}$, $\mathrm{dd}, J=10,2 \mathrm{~Hz})]$, and AX-type methylene proton signals due to $\mathrm{H}_{2}-28[\delta 3.34(1 \mathrm{H}, \mathrm{brd}, J=7 \mathrm{~Hz}), 4.39(1 \mathrm{H}, \mathrm{d}, J=$ $7 \mathrm{~Hz})]$. From the above results and the ${ }^{13} \mathrm{C}-\mathrm{NMR}$ spectrum
(Table I), the aglycone was identified as saikogenin $\mathrm{F}^{2,3)}$ For the purpose of studying the binding site of the three monosaccharides, we carried out a difference nuclear Overhauser effect (NOE) experiment. When the signals at $\delta$ $4.94(1 \mathrm{H}, \mathrm{d}, J=8 \mathrm{~Hz}, \mathrm{H}-1$ of Fuc $), 5.22$ and $5.04(1 \mathrm{H}, \mathrm{d}$, $J=8 \mathrm{~Hz}, \mathrm{H}-1$ of each Glc) were irradiated, NOEs were observed at signals due to the H-3 $[\delta 4.23(1 \mathrm{H}, \mathrm{dd}, J=12$, $5 \mathrm{~Hz})]$ of the aglycone, the $\mathrm{H}-3[\delta 4.12(1 \mathrm{H}, \mathrm{dd}, J=9.5$, $3 \mathrm{~Hz})]$ of the fucose and the $\mathrm{H}_{2}-6[\delta 4.23(1 \mathrm{H}, \mathrm{dd}, J=12$, $6 \mathrm{~Hz}), 4.81(1 \mathrm{H}, \mathrm{brd}, J=12 \mathrm{~Hz})]$ of the glucose attached to the $\mathrm{C}-3$ of the fucose, respectively. Therefore, clinoposapo-
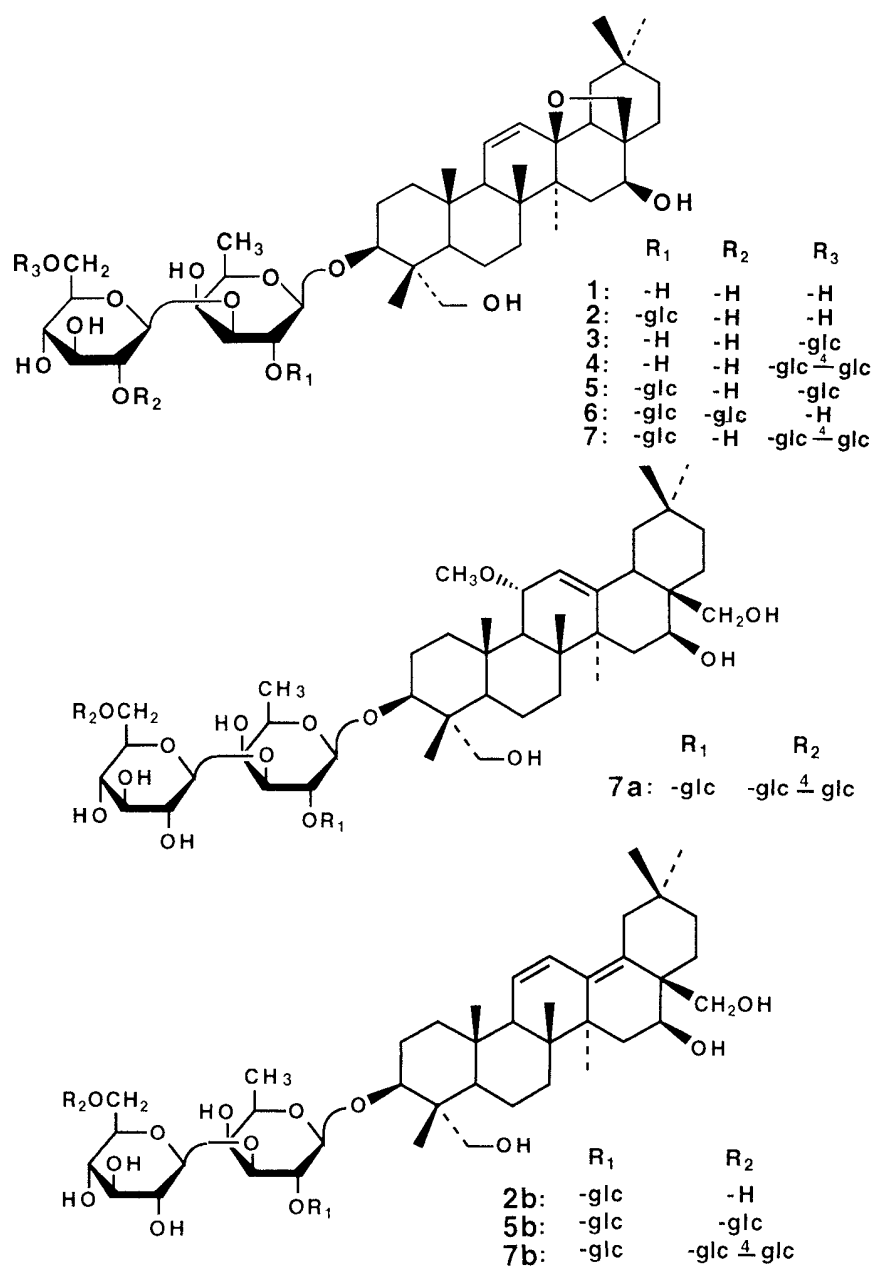

Chart 1 
TABle I. ${ }^{13} \mathrm{C}$-NMR Chemical Shifts of the Aglycone Moiety in Pyridine- $d_{5}$ at $35^{\circ} \mathrm{C}$

\begin{tabular}{|c|c|c|c|c|c|c|c|c|c|c|c|}
\hline & 1 & 2 & 3 & 4 & 5 & 6 & 7 & $7 \mathbf{a}$ & $2 \mathbf{b}$ & $5 b$ & $7 b$ \\
\hline \multicolumn{12}{|l|}{ Aglycone } \\
\hline C- 1 & 38.7 & 38.7 & 38.7 & 38.7 & 38.7 & 38.7 & 38.7 & 40.1 & 38.5 & 38.5 & 38.5 \\
\hline C- 2 & 26.1 & 26.0 & 26.1 & 26.1 & 26.0 & 26.1 & 26.0 & 26.3 & 26.1 & 26.0 & 26.0 \\
\hline C- 3 & 81.7 & 82.6 & 81.8 & 81.8 & 82.8 & 82.2 & 82.8 & 83.1 & 82.7 & 82.8 & 82.8 \\
\hline C- 4 & 43.8 & 43.8 & 43.7 & 43.7 & 43.8 & 43.8 & 43.8 & 43.9 & 43.8 & 43.8 & 43.8 \\
\hline C- 5 & 47.4 & 47.9 & 47.5 & 47.5 & 48.1 & 47.8 & 48.1 & 48.4 & 47.8 & 48.0 & 48.0 \\
\hline C- 6 & 17.6 & 17.7 & 17.6 & 17.6 & 17.7 & 17.7 & 17.7 & 18.4 & 18.3 & 18.3 & 18.4 \\
\hline C- 7 & 31.7 & 31.7 & 31.6 & 31.6 & 31.7 & 31.6 & 31.7 & 33.3 & 32.5 & 32.5 & 32.5 \\
\hline C- 8 & 42.3 & 42.2 & 42.3 & 42.3 & 42.3 & 42.2 & 42.3 & 41.0 & 40.5 & 40.5 & 40.5 \\
\hline C- 9 & 53.2 & 53.1 & 53.2 & 53.2 & 53.1 & 53.1 & 53.1 & 52.2 & 54.6 & 54.6 & 54.6 \\
\hline C- 10 & 36.3 & 36.3 & 36.4 & 36.4 & 36.3 & 36.3 & 36.3 & 38.1 & 36.5 & 36.5 & 36.5 \\
\hline $\mathrm{C}-11$ & 132.2 & 132.2 & 132.2 & 132.2 & 132.2 & 132.2 & 132.2 & 76.0 & 127.1 & 127.1 & 127.1 \\
\hline $\mathrm{C}-12$ & 131.2 & 131.2 & 131.2 & 131.2 & 131.2 & 131.1 & 131.2 & 122.6 & 125.7 & 125.7 & 125.7 \\
\hline C-13 & 84.0 & 84.0 & 84.0 & 84.0 & 84.0 & 84.0 & 84.0 & 148.3 & 136.5 & 136.5 & 136.5 \\
\hline C-14 & 45.7 & 45.7 & 45.7 & 45.7 & 45.7 & 45.7 & 45.7 & 44.0 & 44.3 & 44.3 & 44.3 \\
\hline C- -15 & 36.2 & 36.2 & 36.2 & 36.2 & 36.2 & 36.2 & 36.2 & 36.9 & 35.0 & 35.0 & 35.0 \\
\hline C- 16 & 64.1 & 64.1 & 64.1 & 64.1 & 64.1 & 64.1 & 64.1 & 66.4 & 76.6 & 76.6 & 76.6 \\
\hline C-17 & 47.0 & 47.0 & 47.0 & 47.0 & 47.0 & 47.0 & 47.0 & 43.7 & 44.5 & 44.5 & 44.5 \\
\hline C-18 & 52.2 & 52.2 & 52.2 & 52.2 & 52.2 & 52.2 & 52.2 & 44.1 & 133.4 & 133.4 & 133.4 \\
\hline C- 19 & 37.8 & 37.8 & 37.8 & 37.8 & 37.8 & 37.8 & 37.8 & 47.0 & 38.4 & 38.5 & 38.4 \\
\hline C-20 & 31.6 & 31.6 & 31.6 & 31.6 & 31.6 & 31.6 & 31.6 & 31.1 & 32.7 & 32.7 & 32.7 \\
\hline C-21 & 34.8 & 34.7 & 34.8 & 34.8 & 34.8 & 34.8 & 34.8 & 34.3 & 35.2 & 35.2 & 35.2 \\
\hline $\mathrm{C}-22$ & 25.8 & 25.8 & 25.8 & 25.8 & 25.8 & 25.8 & 25.8 & 26.1 & 30.0 & 30.0 & 30.0 \\
\hline C-23 & 64.2 & 64.7 & 64.2 & 64.3 & 64.9 & 64.5 & 64.9 & 65.3 & 64.7 & 64.8 & 64.9 \\
\hline C-24 & 13.0 & 12.7 & 13.0 & 13.0 & 12.7 & 12.8 & 12.7 & 13.2 & 12.8 & 12.8 & 12.8 \\
\hline C-25 & 18.8 & 18.7 & 18.8 & 18.8 & 18.7 & 18.7 & 18.7 & 17.9 & 18.8 & 18.7 & 18.7 \\
\hline C-26 & 20.1 & 20.0 & 20.1 & 20.1 & 20.1 & 20.0 & 20.1 & 18.4 & 17.1 & 17.1 & 17.1 \\
\hline C-27 & 20.9 & 20.8 & 20.9 & 20.9 & 20.9 & 20.9 & 20.9 & 26.3 & 22.0 & 22.0 & 22.0 \\
\hline C -28 & 73.0 & 73.0 & 73.1 & 73.1 & 73.0 & 73.0 & 73.0 & 68.8 & 64.0 & 64.8 & 64.0 \\
\hline C- 29 & 33.7 & 33.7 & 33.7 & 33.7 & 33.7 & 33.7 & 33.7 & 33.3 & 24.9 & 24.9 & 24.9 \\
\hline C -30 & 23.9 & 23.8 & 23.9 & 23.9 & 23.9 & 23.8 & 23.9 & 24.1 & 32.3 & 32.3 & 32.3 \\
\hline $\mathrm{OMe}$ & & & & & & & & 54.1 & & & \\
\hline
\end{tabular}

Recorded on a JEOL GSX-270 (67.80 MHz).

TABLE II. ${ }^{13} \mathrm{C}-\mathrm{NMR}$ Chemical Shifts of the Sugar Moiety in Pyridine- $d_{5}$ at $35^{\circ} \mathrm{C}$

\begin{tabular}{|c|c|c|c|c|c|c|c|c|c|c|c|}
\hline & 1 & 2 & $2 b$ & 3 & 4 & 5 & $\mathbf{5 b}$ & 6 & 7 & $7 \mathbf{a}$ & $7 \mathrm{~b}$ \\
\hline \multicolumn{12}{|l|}{ Fucose } \\
\hline C-1 & 106.0 & 104.1 & 104.1 & 106.0 & 106.0 & 104.0 & 104.0 & 104.7 & 104.0 & 104.0 & 104.0 \\
\hline $\mathrm{C}-2$ & 71.7 & 77.2 & 77.2 & 71.7 & 71.7 & 77.3 & 77.3 & 76.9 & 77.2 & 77.2 & 77.2 \\
\hline$C-3$ & 85.3 & 84.9 & 84.9 & 85.0 & 85.3 & 85.0 & 85.0 & 80.8 & 85.1 & 85.1 & 85.1 \\
\hline C-4 & 71.9 & 72.0 & 72.0 & 72.1 & 72.1 & 71.9 & 71.9 & 72.5 & 71.9 & 72.0 & 71.9 \\
\hline$C-5$ & 71.1 & 70.5 & 70.5 & 71.1 & 71.1 & 70.5 & 70.6 & 70.7 & 70.6 & 70.6 & 70.6 \\
\hline C-6 & 17.3 & 17.2 & 17.3 & 17.3 & 17.3 & 17.3 & 17.3 & 17.1 & 17.3 & 17.3 & 17.3 \\
\hline \multicolumn{12}{|c|}{ Glucose (at C-2 of fuc) } \\
\hline C-1 & & 104.1 & 104.1 & & & 104.1 & 104.0 & 102.9 & 104.1 & 104.1 & 104.0 \\
\hline $\mathrm{C}-2$ & & 76.2 & 76.3 & & & 76.2 & 76.2 & 76.7 & 76.2 & 76.1 & 76.2 \\
\hline $\mathrm{C}-3$ & & 78.8 & 78.8 & & & 78.8 & 78.8 & 78.2 & 78.8 & 78.8 & 78.8 \\
\hline C-4 & & 72.3 & 72.3 & & & 72.2 & 72.2 & 72.4 & 72.2 & 72.1 & 72.2 \\
\hline C-5 & & 77.5 & 77.5 & & & 77.5 & 77.5 & 77.4 & 77.4 & 77.5 & 77.5 \\
\hline C-6 & & 63.2 & 63.2 & & & 63.1 & 63.2 & 63.3 & 63.1 & 63.1 & 63.2 \\
\hline \multicolumn{12}{|c|}{ Glucose (at C-3 of fuc) } \\
\hline $\mathrm{C}-1$ & 106.7 & 105.2 & 105.2 & 106.2 & 106.3 & 104.8 & 104.9 & 101.7 & 104.9 & 104.9 & 104.9 \\
\hline $\mathrm{C}-2$ & 75.9 & 75.4 & 75.4 & 75.3 & 75.6 & 75.2 & 75.2 & 85.3 & 75.2 & 75.2 & 75.2 \\
\hline C-3 & 78.8 & 78.4 & 78.4 & 78.4 & 78.4 & 78.3 & 78.4 & 77.8 & 78.3 & 78.3 & 78.3 \\
\hline C-4 & 72.2 & 71.7 & 71.7 & 71.8 & 71.8 & 72.0 & 72.0 & 71.3 & 71.9 & 72.0 & 71.9 \\
\hline C-5 & 78.5 & 78.5 & 78.5 & 77.4 & 77.3 & 77.1 & 77.1 & 78.4 & 77.1 & 77.1 & 77.1 \\
\hline C-6 & 62.8 & 62.6 & 62.6 & 70.3 & 70.3 & 70.3 & 70.3 & 62.5 & 70.3 & 70.3 & 70.3 \\
\hline \multicolumn{12}{|c|}{$\begin{array}{l}\text { Glucose (at C-6 of glc at C-3 of fuc) } \\
\text { (6: at C-2 of glc at C-3 of fuc) }\end{array}$} \\
\hline C-1 & & & & 105.5 & 105.1 & 105.4 & 105.4 & 106.4 & 105.0 & 105.0 & 105.0 \\
\hline $\mathrm{C}-2$ & & & & 75.5 & 74.9 & 75.4 & 75.4 & 75.8 & 74.9 & 74.9 & 74.9 \\
\hline C-3 & & & & 78.4 & 76.6 & 78.4 & 78.4 & 78.4 & 76.5 & 76.6 & 76.6 \\
\hline C-4 & & & & 71.7 & 80.9 & 71.6 & 71.7 & 71.6 & 80.9 & 80.9 & 80.9 \\
\hline C-5 & & & & 78.4 & 76.5 & 78.4 & 78.4 & 78.9 & 76.4 & 76.5 & 76.5 \\
\hline C- 6 & & & & 62.8 & 62.0 & 62.7 & 62.7 & 62.9 & 61.9 & 62.0 & 62.0 \\
\hline \multicolumn{12}{|c|}{ Terminal glucose } \\
\hline C-1 & & & & & 104.9 & & & & 104.9 & 104.9 & 104.9 \\
\hline $\mathrm{C}-2$ & & & & & 74.7 & & & & 74.7 & 74.7 & 74.7 \\
\hline $\mathrm{C}-3$ & & & & & 78.3 & & & & 78.2 & 78.2 & 78.2 \\
\hline C-4 & & & & & 71.6 & & & & 71.6 & 71.5 & 71.5 \\
\hline C-5 & & & & & 78.5 & & & & 78.4 & 78.5 & 78.5 \\
\hline C-6 & & & & & 62.5 & & & & 62.4 & 62.5 & 62.4 \\
\hline
\end{tabular}

Recorded on a JEOL GSX-270 (67.80 MHz). 
TABLe III. ${ }^{1} \mathrm{H}-\mathrm{NMR}$ Chemical Shifts of Compounds $\mathbf{2 b}-\mathbf{7}$ in Pyridine- $d_{5}$ at $35^{\circ} \mathrm{C}$

\begin{tabular}{|c|c|c|c|c|c|}
\hline & $\mathbf{2 b}$ & 3 & 4 & 5 & $5 \mathbf{b}$ \\
\hline \multicolumn{6}{|c|}{ Aglycone } \\
\hline H- 3 & $4.15(1 \mathrm{H}, \mathrm{dd}, J=12,5 \mathrm{~Hz})$ & $4.23(1 \mathrm{H}, \mathrm{dd}, J=12,5 \mathrm{~Hz})$ & $4.22(1 \mathrm{H}, \mathrm{dd}, J=12,5 \mathrm{~Hz})$ & $4.07(1 \mathrm{H}, \mathrm{dd}, J=12,5 \mathrm{~Hz})$ & $4.07^{a)}$ \\
\hline \multicolumn{6}{|c|}{ (1) } \\
\hline H-11 & $6.51(1 \mathrm{H}, \mathrm{dd}, J=10,2 \mathrm{~Hz})$ & $6.01(1 \mathrm{H}$, brd, $J=10 \mathrm{~Hz})$ & $6.00(1 \mathrm{H}$, brd, $J=10 \mathrm{~Hz})$ & $5.98(1 \mathrm{H}, \mathrm{brd}, J=10 \mathrm{~Hz})$ & $6.52(1 \mathrm{H}$, brd, $J=10 \mathrm{~Hz})$ \\
\hline $\mathrm{H}-12$ & $5.68(1 \mathrm{H}$, br d, $J=10 \mathrm{~Hz})$ & $5.66(1 \mathrm{H}, \mathrm{dd}, J=10,2 \mathrm{~Hz})$ & $5.66(1 \mathrm{H}, \mathrm{dd}, J=10,2 \mathrm{~Hz})$ & $5.64(1 \mathrm{H}, \mathrm{dd}, J=10,2 \mathrm{~Hz})$ & $5.70(1 \mathrm{H}, \mathrm{brd}, J=10 \mathrm{~Hz})$ \\
\hline H-16 & $4.22^{a)}$ & $4.51(1 \mathrm{H}, \mathrm{m})$ & $4.50(1 \mathrm{H}, \mathrm{m})$ & $4.49(1 \mathrm{H}, \mathrm{m})$ & $4.21(1 \mathrm{H}, \mathrm{m})$ \\
\hline \multirow[t]{2}{*}{$\mathrm{H}-23$} & $3.70(1 \mathrm{H}, \mathrm{d}, J=11 \mathrm{~Hz})$ & $3.68(1 \mathrm{H}, \mathrm{d}, J=11 \mathrm{~Hz})$ & $3.68(1 \mathrm{H}, \mathrm{d}, J=11 \mathrm{~Hz})$ & $3.70(1 \mathrm{H}, \mathrm{d}, J=11 \mathrm{~Hz})$ & $3.69(1 \mathrm{H}, \mathrm{d}, J=11 \mathrm{~Hz})$ \\
\hline & $4.37(1 \mathrm{H}, \mathrm{d}, J=11 \mathrm{~Hz})$ & $4.32(1 \mathrm{H}, \mathrm{d}, J=11 \mathrm{~Hz})$ & $4.32(1 \mathrm{H}, \mathrm{d}, J=11 \mathrm{~Hz})$ & $4.32^{a)}$ & $4.35(1 \mathrm{H}, \mathrm{d}, J=11 \mathrm{~Hz})$ \\
\hline $\mathrm{H}-24$ & $1.05(3 \mathrm{H}, \mathrm{s})$ & $0.92(3 \mathrm{H}, \mathrm{s})$ & $0.90(3 \mathrm{H}, \mathrm{s})$ & $1.06(3 \mathrm{H}, \mathrm{s})$ & $1.06(3 \mathrm{H}, \mathrm{s})$ \\
\hline $\mathrm{H}-25$ & $0.95(3 \mathrm{H}, \mathrm{s})$ & $1.00(3 \mathrm{H}, \mathrm{s})$ & $1.00(3 \mathrm{H}, \mathrm{s})$ & $0.97(3 \mathrm{H}, \mathrm{s})$ & $0.96(3 \mathrm{H}, \mathrm{s})$ \\
\hline $\mathrm{H}-26$ & $0.85(3 \mathrm{H}, \mathrm{s})$ & $1.39(3 \mathrm{H}, \mathrm{s})$ & $1.39(3 \mathrm{H}, \mathrm{s})$ & $1.38(3 \mathrm{H}, \mathrm{s})$ & $0.85(3 \mathrm{H}, \mathrm{s})$ \\
\hline $\mathrm{H}-27$ & $1.07(3 \mathrm{H}, \mathrm{s})$ & $1.11(3 \mathrm{H}, \mathrm{s})$ & $1.11(3 \mathrm{H}, \mathrm{s})$ & $1.11(3 \mathrm{H}, \mathrm{s})$ & $1.08(3 \mathrm{H}, \mathrm{s})$ \\
\hline \multirow[t]{2}{*}{ H-28 } & $4.18^{a)}$ & $3.34(1 \mathrm{H}, \mathrm{brd}, J=7 \mathrm{~Hz})$ & $3.33(1 \mathrm{H}, \mathrm{brd}, J=7 \mathrm{~Hz})$ & $3.33(1 \mathrm{H}$, br d, $J=7 \mathrm{~Hz})$ & \\
\hline & $4.20^{a)}$ & $4.39(1 \mathrm{H}, \mathrm{d}, J=7 \mathrm{~Hz})$ & $4.38(1 \mathrm{H}, \mathrm{d}, J=7 \mathrm{~Hz})$ & $4.37(1 \mathrm{H}, \mathrm{d}, J=7 \mathrm{~Hz})$ & \\
\hline H-29 & $0.87(3 \mathrm{H}, \mathrm{s})$ & $0.94(3 \mathrm{H}, \mathrm{s})$ & $0.94(3 \mathrm{H}, \mathrm{s})$ & $0.94(3 \mathrm{H}, \mathrm{s})$ & $0.87(3 \mathrm{H}, \mathrm{s})$ \\
\hline $\mathrm{H}-30$ & $0.97(3 \mathrm{H}, \mathrm{s})$ & $0.91(3 \mathrm{H}, \mathrm{s})$ & $0.90(3 \mathrm{H}, \mathrm{s})$ & $0.91(3 \mathrm{H}, \mathrm{s})$ & $0.98(3 \mathrm{H}, \mathrm{s})$ \\
\hline \multicolumn{6}{|c|}{$\mathrm{H}-\mathrm{OMe}$} \\
\hline \multicolumn{6}{|c|}{ Fucose } \\
\hline $\mathrm{H}-1$ & $4.92(1 \mathrm{H}, \mathrm{d}, J=8 \mathrm{~Hz})$ & $4.94(1 \mathrm{H}, \mathrm{d}, J=8 \mathrm{~Hz})$ & $4.93(1 \mathrm{H}, \mathrm{d}, J=8 \mathrm{~Hz})$ & $4.88(1 \mathrm{H}, \mathrm{d}, J=8 \mathrm{~Hz})$ & $4.91(1 \mathrm{H}, \mathrm{d}, J=8 \mathrm{~Hz})$ \\
\hline $\mathrm{H}-2$ & $4.64(1 \mathrm{H}, \mathrm{dd}, J=9,8 \mathrm{~Hz})$ & $4.49(1 \mathrm{H}, \mathrm{dd}, J=9,8.5 \mathrm{~Hz})$ & $4.49^{a)}$ & $4.62(1 \mathrm{H}, \mathrm{dd}, J=10,8.5 \mathrm{~Hz})$ & $4.63(1 \mathrm{H}, \mathrm{dd}, J=9.5,8 \mathrm{~Hz})$ \\
\hline H- 3 & $4.09(1 \mathrm{H}, \mathrm{dd}, J=9,3.5 \mathrm{~Hz})$ & $4.12(1 \mathrm{H}, \mathrm{dd}, J=9.5,3 \mathrm{~Hz})$ & $4.10(1 \mathrm{H}, \mathrm{dd}, J=9.5,3 \mathrm{~Hz})$ & $4.14^{a)}$ & $4.14^{a)}$ \\
\hline H- 4 & $4.25(1 \mathrm{H}, \mathrm{d}, J=3.5 \mathrm{~Hz})$ & $4.33(1 \mathrm{H}, \mathrm{d}, J=3 \mathrm{~Hz})$ & $4.33^{a)}$ & $4.39(1 \mathrm{H}, \mathrm{brs})$ & $4.39(1 \mathrm{H}, \mathrm{d}, J=3 \mathrm{~Hz})$ \\
\hline H- 5 & $3.63(1 \mathrm{H}, \mathrm{m})$ & $3.86(1 \mathrm{H}, \mathrm{m})$ & $3.86(1 \mathrm{H}, \mathrm{m})$ & $3.84(1 \mathrm{H}, \mathrm{m})$ & $3.86(1 \mathrm{H}, \mathrm{m})$ \\
\hline H- 6 & $1.41(3 \mathrm{H}, \mathrm{d}, J=6 \mathrm{~Hz})$ & $1.51(3 \mathrm{H}, \mathrm{d}, J=6 \mathrm{~Hz})$ & $1.52(3 \mathrm{H}, \mathrm{d}, J=6 \mathrm{~Hz})$ & $1.54(3 \mathrm{H}, \mathrm{d}, J=6 \mathrm{~Hz})$ & $1.55(3 \mathrm{H}, \mathrm{d}, J=6 \mathrm{~Hz})$ \\
\hline \multicolumn{6}{|c|}{ Glucose (at C-2 of fuc) } \\
\hline H- 1 & $5.56(1 \mathrm{H}, \mathrm{d}, J=8 \mathrm{~Hz})$ & & & $5.51(1 \mathrm{H}, \mathrm{d}, J=8 \mathrm{~Hz})$ & $5.52(1 \mathrm{H}, \mathrm{d}, J=8 \mathrm{~Hz})$ \\
\hline \multicolumn{6}{|c|}{ Glucose (at C-3 of fuc) } \\
\hline H- 1 & $5.28(1 \mathrm{H}, \mathrm{d}, J=8 \mathrm{~Hz})$ & $5.22(1 \mathrm{H}, \mathrm{d}, J=8 \mathrm{~Hz})$ & $5.20(1 \mathrm{H}, \mathrm{d}, J=8 \mathrm{~Hz})$ & $5.15(1 \mathrm{H}, \mathrm{d}, J=8 \mathrm{~Hz})$ & $5.16(1 \mathrm{H}, \mathrm{d}, J=8 \mathrm{~Hz})$ \\
\hline H. 2 & $3.98(1 \mathrm{H}, \mathrm{t}, J=8.5 \mathrm{~Hz})$ & $3.97(1 \mathrm{H}, \mathrm{t}, J=8.5 \mathrm{~Hz})$ & $3.96(1 \mathrm{H}, \mathrm{t}, J=8 \mathrm{~Hz})$ & $3.94(1 \mathrm{H}, \mathrm{t}, J=8.5 \mathrm{~Hz})$ & $3.94(1 \mathrm{H}, \mathrm{t}, J=8.5 \mathrm{~Hz})$ \\
\hline H- 3 & $4.22^{a)}$ & $4.17(1 \mathrm{H}, \mathrm{t}, J=8.5 \mathrm{~Hz})$ & $4.16(1 \mathrm{H}, \mathrm{t}, J=8.5 \mathrm{~Hz})$ & $4.13^{a)}$ & \\
\hline H- 4 & $4.20^{a)}$ & $4.02(1 \mathrm{H}, \mathrm{t}, J=8.5 \mathrm{~Hz})$ & $4.03^{a)}$ & $3.94^{a)}$ & $3.94(1 \mathrm{H}, \mathrm{t}, J=8.5 \mathrm{~Hz})$ \\
\hline H- 5 & $3.93(1 \mathrm{H}, \mathrm{m})$ & $4.09(1 \mathrm{H}, \mathrm{m})$ & $4.07^{a)}$ & $4.04(1 \mathrm{H}, \mathrm{m})$ & $4.06(1 \mathrm{H}, \mathrm{m})$ \\
\hline \multirow{2}{*}{ H- 6} & $4.30(1 \mathrm{H}, \mathrm{dd}, J=12,6 \mathrm{~Hz})$ & $4.23(1 \mathrm{H}, \mathrm{dd}, J=12,6 \mathrm{~Hz})$ & $4.20^{a)}$ & $4.13^{a)}$ & \\
\hline & $4.44(1 \mathrm{H}, \mathrm{dd}, J=12,2 \mathrm{~Hz})$ & $4.81(1 \mathrm{H}$, brd, $J=12 \mathrm{~Hz})$ & $4.77(1 \mathrm{H}$, brd, $J=12 \mathrm{~Hz})$ & $4.76(1 \mathrm{H}, \mathrm{brd}, J=12 \mathrm{~Hz})$ & $4.78(1 \mathrm{H}, \mathrm{brd}, J=12 \mathrm{~Hz})$ \\
\hline \multicolumn{6}{|c|}{ Glucose (at C-6 of glc at C-3 of fuc) } \\
\hline \multicolumn{2}{|l|}{$\mathrm{H}-1$} & $5.04(1 \mathrm{H}, \mathrm{d}, J=8 \mathrm{~Hz})$ & $4.98(1 \mathrm{H}, \mathrm{d}, J=8 \mathrm{~Hz})$ & $4.95(1 \mathrm{H}, \mathrm{d}, J=8 \mathrm{~Hz})$ & $4.96(1 \mathrm{H}, \mathrm{d}, J=8 \mathrm{~Hz})$ \\
\hline \multicolumn{2}{|l|}{ H- 2} & $4.00(1 \mathrm{H}, \mathrm{t}, J=8.5 \mathrm{~Hz})$ & $3.99(1 \mathrm{H}, \mathrm{t}, J=8 \mathrm{~Hz})$ & $3.97(1 \mathrm{H}, \mathrm{t}, J=8.5 \mathrm{~Hz})$ & $3.98(1 \mathrm{H}, \mathrm{t}, J=8.5 \mathrm{~Hz})$ \\
\hline \multicolumn{2}{|l|}{ H- 3} & $4.17^{a)}$ & $4.19(1 \mathrm{H}, \mathrm{t}, J=8.5 \mathrm{~Hz})$ & $4.17^{a)}$ & \\
\hline \multicolumn{2}{|l|}{ H- 4} & $4.21^{a)}$ & $4.30(1 \mathrm{H}, \mathrm{t}, J=8.5 \mathrm{~Hz})$ & $4.19(1 \mathrm{H}, \mathrm{t}, J=9 \mathrm{~Hz})$ & \\
\hline \multicolumn{2}{|l|}{ H. 5} & $3.85(1 \mathrm{H}, \mathrm{m})$ & $3.79(1 \mathrm{H}, \mathrm{m})$ & $3.84(1 \mathrm{H}, \mathrm{m})$ & $3.86(1 \mathrm{H}, \mathrm{m})$ \\
\hline \multirow{2}{*}{\multicolumn{2}{|c|}{ H- 6}} & $4.23^{a)}$ & $4.39^{a)}$ & $4.32^{a)}$ & $4.32(1 \mathrm{H}, \mathrm{dd}, J=12,6 \mathrm{~Hz})$ \\
\hline & & $4.46(1 \mathrm{H}, \mathrm{dd}, J=12,2 \mathrm{~Hz})$ & $4.48^{a)}$ & $4.46(1 \mathrm{H}$, brd, $J=12 \mathrm{~Hz})$ & $4.47(1 \mathrm{H}, \mathrm{dd}, J=12,2 \mathrm{~Hz})$ \\
\hline \multicolumn{6}{|c|}{ Terminal glucose } \\
\hline \multicolumn{3}{|l|}{ H- 1} & $5.14(1 \mathrm{H}, \mathrm{d}, J=8 \mathrm{~Hz})$ & & \\
\hline
\end{tabular}

Recorded on a JEOL GSX-500 (500 MHz). a) Overlapped with other signals.

nin I can be assigned the structure 3, shown in Chart 1.

Clinoposaponins II (4), III (5) and IV (6) gave the same FAB-MS $\left(m / z 1128[\mathrm{M}+\mathrm{Na}]^{+}\right)$and elemental analysis data. Mild methanolysis and subsequent acid hydrolysis of 4, 5 and 6 yielded saikogenin $A$ as an aglycone and fucose and glucose in the ratio of $1: 3$ as a sugar moiety. The ${ }^{1} \mathrm{H}$ - and ${ }^{13} \mathrm{C}$-NMR spectra of 4 resembled those of 3 , and those of 5 resembled 2 except for the presence of one more glucose. When the ${ }^{13} \mathrm{C}-\mathrm{NMR}$ spectrum of $\mathbf{4}$ was compared with that of 3, glycosylation shifts were observed at C-3 $(-1.8 \mathrm{ppm}), \mathrm{C}-4(+9.2 \mathrm{ppm})$ and C-5 $(-1.9 \mathrm{ppm})$ of glucose attached to the $\mathrm{C}-6$ of the inner glucose. When the signal at $\delta 5.14(1 \mathrm{H}, \mathrm{d}, J=8 \mathrm{~Hz}, \mathrm{H}-1$ of Glc) was irradiated, NOE was observed at a signal due to the H-4 of glucose attached to the C-6 of the inner glucose. In the same way, the ${ }^{13} \mathrm{C}-\mathrm{NMR}$ spectrum of $\mathbf{5}$ was compared with that of $\mathbf{2}$, glycosylation shifts were observed at C-5 $(-1.4 \mathrm{ppm})$ and C-6 $(+7.7 \mathrm{ppm})$ of glucose attached to the C-3 of the fucose. When the signal at $\delta 4.95(1 \mathrm{H}, \mathrm{d}, J=8 \mathrm{~Hz}, \mathrm{H}-1$ of Glc) was irradiated, NOE was observed at a signal due to the $\mathrm{H}_{2}-6$ of glucose attached to the $\mathrm{C}-3$ of the fucose. According to the glycosylation shifts and the NOE experiment, one more glucose was attached to the $\mathrm{C}-4$ of the glucose attached to the C-6 of the inner glucose in 4 , and to the C- 6 of the glucose attached to the C-3 of the fucose in 5. Therefore, clinoposaponins II and III can be formulated as $\mathbf{4}$ and $\mathbf{5}$, respectively, in Chart 1 . On the other hand, the ${ }^{1} \mathrm{H}$ - and ${ }^{13} \mathrm{C}-\mathrm{NMR}$ data of sugar moiety of 6 were not the same as those of $\mathbf{4}$ and $\mathbf{5}$. Therefore, the binding site of the four monosaccharides were determined by the NOE experiment. When the signals at $\delta 4.85$ (the $\mathrm{H}-1$ of fucose), $5.80,5.82$ and 5.26 (the $\mathrm{H}-1$ of each glucose) were irradiated, NOEs were observed at signals due to the $\mathrm{H}-3$ of the aglycone, the $\mathrm{H}-2$ of the fucose, the $\mathrm{H}-3$ of the fucose and the $\mathrm{H}-2$ of the glucose attached to the $\mathrm{C}-3$ of the fucose, respectively. Based on the above evidence, the structure of clinoposaponin IV was assigned as $\mathbf{6}$ in Chart 1.

Clinoposaponin V (7) revealed an $[\mathrm{M}+\mathrm{Na}]^{+}$ion peak at $m / z 1290$ in the FAB-MS and elemental analysis data was consistent with the moleculr formula $\mathrm{C}_{60} \mathrm{H}_{98} \mathrm{O}_{28}$. $7 \mathrm{H}_{2} \mathrm{O}$. Mild methanolysis of 7 afforded saikogenin $\mathrm{A}$, and subsequent acid hydrolysis of the methanolysate yielded fucose and glucose in the ratio of $1: 4$. The ${ }^{1} \mathrm{H}$ - and ${ }^{13} \mathrm{C}$-NMR data of 7 were similar to those of 5 except for the presence of one more glucose. When the ${ }^{13} \mathrm{C}-\mathrm{NMR}$ spectrum of 7 was compared with that of $\mathbf{5}$, glycosylation 
TABLE III. (continued)

\begin{tabular}{|c|c|c|c|c|}
\hline & 6 & 7 & $7 \mathbf{a}$ & $7 \mathbf{b}$ \\
\hline \multicolumn{5}{|l|}{ Aglycone } \\
\hline $\mathrm{H}-3$ & $4.12(1 \mathrm{H}, \mathrm{dd}, J=12,5 \mathrm{~Hz})$ & $4.07(1 \mathrm{H}, \mathrm{dd}, J=12,5 \mathrm{~Hz})$ & & \\
\hline H- 9 & & & $1.51(1 \mathrm{H}, \mathrm{d}, J=8 \mathrm{~Hz})$ & \\
\hline $\mathrm{H}-11$ & $5.98(1 \mathrm{H}, \mathrm{brd}, J=10 \mathrm{~Hz})$ & $5.96(1 \mathrm{H}, \mathrm{d}, J=10 \mathrm{~Hz})$ & $3.80(1 \mathrm{H}, \mathrm{dd}, J=8,3 \mathrm{~Hz})$ & $6.52(1 \mathrm{H}, \mathrm{dd}, J=10,2 \mathrm{~Hz})$ \\
\hline $\mathrm{H}-12$ & $5.64(1 \mathrm{H}, \mathrm{dd}, J=10,3 \mathrm{~Hz})$ & $5.62(1 \mathrm{H}, \mathrm{dd}, J=10,2 \mathrm{~Hz})$ & $5.52(1 \mathrm{H}, \mathrm{d}, J=3 \mathrm{~Hz})$ & $5.69(1 \mathrm{H}$, brd, $J=10 \mathrm{~Hz})$ \\
\hline $\mathrm{H}-16$ & $4.49(1 \mathrm{H}, \mathrm{m})$ & $4.50(1 \mathrm{H}, \mathrm{m})$ & & \\
\hline \multirow[t]{2}{*}{$\mathrm{H}-23$} & $3.69(1 \mathrm{H}, \mathrm{d}, J=11 \mathrm{~Hz})$ & $3.70(1 \mathrm{H}, \mathrm{d}, J=11 \mathrm{~Hz})$ & $3.70(1 \mathrm{H}, \mathrm{d}, J=11 \mathrm{~Hz})$ & $3.69(1 \mathrm{H}, \mathrm{d}, J=11 \mathrm{~Hz})$ \\
\hline & $4.34(1 \mathrm{H}, \mathrm{d}, J=11 \mathrm{~Hz})$ & $4.32(1 \mathrm{H}, \mathrm{d}, J=11 \mathrm{~Hz})$ & $4.32(1 \mathrm{H}, \mathrm{d}, J=11 \mathrm{~Hz})$ & $4.35(1 \mathrm{H}, \mathrm{d}, J=11 \mathrm{~Hz})$ \\
\hline $\mathrm{H}-24$ & $0.98(3 \mathrm{H}, \mathrm{s})$ & $1.04(3 \mathrm{H}, \mathrm{s})$ & $1.10(3 \mathrm{H}, \mathrm{s})$ & $1.06(3 \mathrm{H}, \mathrm{s})$ \\
\hline $\mathrm{H}-25$ & $0.97(3 \mathrm{H}, \mathrm{s})$ & $0.95(3 \mathrm{H}, \mathrm{s})$ & $1.09(3 \mathrm{H}, \mathrm{s})$ & $0.95(3 \mathrm{H}, \mathrm{s})$ \\
\hline H-26 & $1.39(3 \mathrm{H}, \mathrm{s})$ & $1.36(3 \mathrm{H}, \mathrm{s})$ & $1.06(3 \mathrm{H}, \mathrm{s})$ & $0.85(3 \mathrm{H}, \mathrm{s})$ \\
\hline $\mathrm{H}-27$ & $1.09(3 \mathrm{H}, \mathrm{s})$ & $1.08(3 \mathrm{H}, \mathrm{s})$ & $1.39(3 \mathrm{H}, \mathrm{s})$ & $1.08(3 \mathrm{H}, \mathrm{s})$ \\
\hline \multirow[t]{2}{*}{ H-28 } & $3.32(1 \mathrm{H}$, brd, $J=7 \mathrm{~Hz})$ & $3.33(1 \mathrm{H}$, brd,$J=7 \mathrm{~Hz})$ & & \\
\hline & $4.37(1 \mathrm{H}, \mathrm{d}, J=7 \mathrm{~Hz})$ & $4.38(1 \mathrm{H}, \mathrm{d}, J=7 \mathrm{~Hz})$ & & \\
\hline H-29 & $0.93(3 \mathrm{H}, \mathrm{s})$ & $0.92(3 \mathrm{H}, \mathrm{s})$ & $0.90(3 \mathrm{H}, \mathrm{s})$ & $0.87(3 \mathrm{H}, \mathrm{s})$ \\
\hline $\mathrm{H}-30$ & $0.90(3 \mathrm{H}, \mathrm{s})$ & $0.88(3 \mathrm{H}, \mathrm{s})$ & $1.00(3 \mathrm{H}, \mathrm{s})$ & $0.98(3 \mathrm{H}, \mathrm{s})$ \\
\hline $\mathrm{H}-\mathrm{OMe}$ & & & $3.23(3 \mathrm{H}, \mathrm{s})$ & \\
\hline \multicolumn{5}{|c|}{ Fucose } \\
\hline $\mathrm{H}-\mathrm{l}$ & $4.85(1 \mathrm{H}, \mathrm{d}, J=8 \mathrm{~Hz})$ & $4.89(1 \mathrm{H}, \mathrm{d}, J=8 \mathrm{~Hz})$ & $4.88(1 \mathrm{H}, \mathrm{d}, J=8 \mathrm{~Hz})$ & $4.91(1 \mathrm{H}, \mathrm{d}, J=8 \mathrm{~Hz})$ \\
\hline H- 2 & $4.94(1 \mathrm{H}, \mathrm{dd}, J=9,8 \mathrm{~Hz})$ & $4.62(1 \mathrm{H}, \mathrm{dd}, J=9.5,8 \mathrm{~Hz})$ & $4.63^{a)}$ & $4.64(1 \mathrm{H}, \mathrm{dd}, J=9.5,8 \mathrm{~Hz})$ \\
\hline H- 3 & $4.28(1 \mathrm{H}, \mathrm{dd}, J=9,3 \mathrm{~Hz})$ & $4.14(1 \mathrm{H}, \mathrm{dd}, J=9.5,3 \mathrm{~Hz})$ & $4.15^{a)}$ & $4.15^{a)}$ \\
\hline H- 4 & $4.08(1 \mathrm{H}, \mathrm{d}, J=3 \mathrm{~Hz})$ & $4.39(1 \mathrm{H}$, brs $)$ & $4.38(1 \mathrm{H}, \mathrm{brs})$ & $4.39(1 \mathrm{H}$, br s $)$ \\
\hline H- 5 & $3.49(1 \mathrm{H}, \mathrm{m})$ & $3.84(1 \mathrm{H}, \mathrm{m})$ & $3.86(1 \mathrm{H}, \mathrm{m})$ & $3.85(1 \mathrm{H}, \mathrm{m})$ \\
\hline H- 6 & $1.34(3 \mathrm{H}, \mathrm{d}, J=6 \mathrm{~Hz})$ & $1.52(3 \mathrm{H}, \mathrm{d}, J=6 \mathrm{~Hz})$ & $1.52(3 \mathrm{H}, \mathrm{d}, J=6 \mathrm{~Hz})$ & $1.56(1 \mathrm{H}, \mathrm{d}, J=6 \mathrm{~Hz})$ \\
\hline \multicolumn{5}{|c|}{ Glucose (at C-2 of fuc) } \\
\hline H- 1 & $5.80(1 \mathrm{H}, \mathrm{d}, J=8 \mathrm{~Hz})$ & $5.51(1 \mathrm{H}, \mathrm{d}, J=8 \mathrm{~Hz})$ & $5.52(1 \mathrm{H}, \mathrm{d}, J=8 \mathrm{~Hz})$ & $5.53(1 \mathrm{H}, \mathrm{d}, J=8 \mathrm{~Hz})$ \\
\hline \multicolumn{5}{|c|}{ Glucose (at C-3 of fuc) } \\
\hline $\mathrm{H}-1$ & $5.82(1 \mathrm{H}, \mathrm{d}, J=8 \mathrm{~Hz})$ & $5.14(1 \mathrm{H}, \mathrm{d}, J=8 \mathrm{~Hz})$ & $5.15(1 \mathrm{H}, \mathrm{d}, J=8 \mathrm{~Hz})$ & $5.15(1 \mathrm{H}, \mathrm{d}, J=8 \mathrm{~Hz})$ \\
\hline $\mathrm{H}-2$ & $4.04(1 \mathrm{H}, \mathrm{t}, J=8.5 \mathrm{~Hz})$ & $3.93(1 \mathrm{H}, \mathrm{t}, J=8.5 \mathrm{~Hz})$ & $3.94(1 \mathrm{H}, \mathrm{t}, J=8 \mathrm{~Hz})$ & \\
\hline $\mathrm{H}-3$ & $4.40(1 \mathrm{H}, \mathrm{t}, J=8.5 \mathrm{~Hz})$ & $4.12^{a)}$ & & \\
\hline $\mathrm{H}-4$ & $4.21(1 \mathrm{H}, \mathrm{t}, J=9 \mathrm{~Hz})$ & $3.94^{a)}$ & & \\
\hline $\mathrm{H}-5$ & $3.96(1 \mathrm{H}, \mathrm{m})$ & $4.03(1 \mathrm{H}, \mathrm{m})$ & $4.03(1 \mathrm{H}, \mathrm{m})$ & \\
\hline \multirow[t]{2}{*}{ H- 6} & $4.33^{a)}$ & $4.10^{a)}$ & $4.09^{a)}$ & \\
\hline & $4.43(1 \mathrm{H}$, br d, $J=12 \mathrm{~Hz})$ & $4.72(1 \mathrm{H}, \mathrm{brd}, J=12 \mathrm{~Hz})$ & $4.73(1 \mathrm{H}$, br d, $J=12 \mathrm{~Hz})$ & $4.73(1 \mathrm{H}$, brd, $J=12 \mathrm{~Hz})$ \\
\hline \multicolumn{2}{|c|}{ Glucose (at $\mathrm{C}-2$ of glc at $\mathrm{C}-3$ of fuc) } & (at C-6 of glc at C-3 of fuc) & & \\
\hline $\mathrm{H}-1$ & $5.26(1 \mathrm{H}, \mathrm{d}, J=8 \mathrm{~Hz})$ & $4.91(1 \mathrm{H}, \mathrm{d}, J=8 \mathrm{~Hz})$ & $4.91(1 \mathrm{H}, \mathrm{d}, J=8 \mathrm{~Hz})$ & $4.92(1 \mathrm{H}, \mathrm{d}, J=8 \mathrm{~Hz})$ \\
\hline H. 2 & $4.32(1 \mathrm{H}, \mathrm{t}, J=8.5 \mathrm{~Hz})$ & $3.96^{a)}$ & $3.97(1 \mathrm{H}, \mathrm{t}, J=8.5 \mathrm{~Hz})$ & \\
\hline H- 3 & $4.11^{a)}$ & $4.20(1 \mathrm{H}, \mathrm{t}, J=8.5 \mathrm{~Hz})$ & $4.20(1 \mathrm{H}, \mathrm{t}, J=8.5 \mathrm{~Hz})$ & $4.20(1 \mathrm{H}, \mathrm{t}, J=8.5 \mathrm{~Hz})$ \\
\hline H- 4 & $4.19(1 \mathrm{H}, \mathrm{t}, J=9 \mathrm{~Hz})$ & $4.30(1 \mathrm{H}, \mathrm{t}, J=9 \mathrm{~Hz})$ & $4.30(1 \mathrm{H}, \mathrm{t}, J=9 \mathrm{~Hz})$ & $4.30(1 \mathrm{H}, \mathrm{t}, J=9 \mathrm{~Hz})$ \\
\hline H- 5 & $3.94(1 \mathrm{H}, \mathrm{m})$ & $3.79(1 \mathrm{H}, \mathrm{m})$ & $3.78(1 \mathrm{H}, \mathrm{m})$ & $3.80(1 \mathrm{H}, \mathrm{m})$ \\
\hline \multirow[t]{2}{*}{ H. 6} & $4.33^{a)}$ & $4.38^{a)}$ & $4.37^{a)}$ & \\
\hline & $4.46(1 \mathrm{H}, \mathrm{brd}, J=12 \mathrm{~Hz})$ & $4.48^{a)}$ & $4.48(1 \mathrm{H}, \mathrm{dd}, J=12,2 \mathrm{~Hz})$ & \\
\hline \multicolumn{5}{|c|}{ Terminal glucose } \\
\hline $\mathrm{H}-1$ & & $5.15(1 \mathrm{H}, \mathrm{d}, J=8 \mathrm{~Hz})$ & $5.15(1 \mathrm{H}, \mathrm{d}, J=8 \mathrm{~Hz})$ & $5.15(1 \mathrm{H}, \mathrm{d}, J=8 \mathrm{~Hz})$ \\
\hline
\end{tabular}

shifts were observed at C-3 $(-1.9 \mathrm{ppm}), \mathrm{C}-4(+9.3 \mathrm{ppm})$ and $\mathrm{C}-5(-2.0 \mathrm{ppm})$ of the glucose attached to the C-6 of the inner glucose. In addition, when the signal at $\delta 5.15$ (the $\mathrm{H}-1$ of the additional glucose) was irradiated, NOE was observed at the signal due to the $\mathrm{H}-4$ of the glucose attached to the C-6 of the inner glucose. Based on the above data, the structure of clinoposaponin $\mathrm{V}$ was assigned as 7 in Chart 1.

We believe that buddlejasaponin IVb (2b), clinoposaponins IIIb (5b), Va (7a) and Vb (7b) were obtained as artifacts of buddlejasaponin IV (2), clinoposaponins III (5) and V (7), respectively. ${ }^{2,3)}$ The ${ }^{1} \mathrm{H}$-NMR spectrum of 7a showed one methoxyl signal $[\delta 3.23(3 \mathrm{H}, \mathrm{s})]$ and one olefinic proton signal $[\delta 5.52(1 \mathrm{H}, \mathrm{d}, J=3 \mathrm{~Hz})]$, and the ${ }^{13} \mathrm{C}$-NMR data of the aglycone moiety of $7 \mathrm{a}$ were very similar to those of saikosaponin $b_{3}{ }^{2,4)}$ On the other hand, the ${ }^{13} \mathrm{C}$-NMR data of the aglycone moiety of $\mathbf{2 b}, \mathbf{5 b}$ and $\mathbf{7 b}$ showed four olefinic carbon signals $(\delta 125.7,127.1,133.4$ and 136.5) and were similar to saikogenin $\mathrm{A}$. Therefore, the structures of buddlejasaponin IVb, clinoposaponins IIIb, $\mathrm{Va}$ and $\mathrm{Vb}$ were assigned as $\mathbf{2 b}, \mathbf{5 b}, \mathbf{7} \mathbf{a}$ and $\mathbf{7 b}$, respectively, in Chart 1.

Clinoposaponins I-V (3-7) were composed of saiko- saponin a and more glucoses, and the sugar linkages of these compounds differed from those of saikosaponins and buddlejasaponins. ${ }^{1)}$

Recently, clinopodiside A was isolated from C. polycephalum C. T. WU et Hsuan by Xue et al. ${ }^{5)}$ Its structure, composed of saikogenin A and three glucoses, is $3 \beta$ $[\beta$-D-glucopyranosyl $(1 \rightarrow 6)$ - $[\beta$-D-glucopyranosyl $(1 \rightarrow 4)]$ - $\beta$ D-glucopyranosyloxy]olean-11,13(18)-diene-16 $\beta, 23,28$-triol. Compared with the clinoposaponins, clinopodiside $\mathrm{A}$ has a diene-type aglycone and no fucose in the sugar moiety.

It is anticipated that $C$. gracile could be a new source of medicinal compounds because it is widespread in EastAsia.

\section{Experimental}

General Procedures ${ }^{1} \mathrm{H}$ - and ${ }^{13} \mathrm{C}-\mathrm{NMR}$ spectra were obtained with a JEOL GSX-270 and GSX-500 FT-NMR, and chemical shifts were given in ppm with tetramethylsilane as the internal standard. FAB-MS were recorded on a JEOL JMS-SX102 mass spectrometer. Optical rotations were measured with a JASCO DIP-360 digital polarimeter. Gas chromatography (GC) was run on a Hitachi G-3000 Gas Chromatograph. Preparative and semi-preparative HPLC separations were carried out on a column of Develosil Lop-ODS $(50 \mathrm{~mm} \times 50 \mathrm{~cm})$ and YMC R-ODS-5 $\mathrm{AQ}(20 \mathrm{~mm} \times 25 \mathrm{~cm})$, respectively. 
Extraction and Isolation $C$. gracile was collected in Shizuoka, Japan in May, 1992. The dried whole plants $(c a .1 .1 \mathrm{~kg})$ were extracted twice with hot water. The extract was passed through a Mitsubishi Diaion HP-20 column. Then the column was washed with water and the absorbed materials were eluated with $50 \%$ aqueous methanol (yield $43 \mathrm{~g}$ ) followed by methanol (yield $15 \mathrm{~g}$ ). The methanol eluate $(2 \mathrm{~g})$ was chromatographed on preparative HPLC equipment [Develosil Lop-ODS, $50 \mathrm{~mm} \times 50 \mathrm{~cm} \times 2$, MeCN-water $(30: 70)-(40: 60)$ linear gradient] to give 23 fractions identified as frs. A-W. Fration $\mathrm{S}$ is a pure compound $2(551 \mathrm{mg})$. From fr. U (75 mg) compounds 1 (17 mg) and $2 \mathbf{b}(34 \mathrm{mg})$ were obtained, from fr. $\mathrm{O}(51 \mathrm{mg})$ compound $5(17 \mathrm{mg})$ was obtained, from fr. $\mathrm{L}(83 \mathrm{mg})$ compounds 7 (34 mg) and $7 \mathbf{a}(9 \mathrm{mg})$ were obtained, from fr. R (47 mg) compounds $3(3 \mathrm{mg}), 6(4 \mathrm{mg}), 2(4 \mathrm{mg})$ and $5 \mathrm{~b}(2 \mathrm{mg})$ were obtained, from fr. P (42 mg) compounds $4(5 \mathrm{mg})$ and $7 \mathrm{~b}(6 \mathrm{mg})$ were obtained. All were isolated by preparative [Develosil Lop-ODS, $50 \mathrm{~mm} \times 50 \mathrm{~cm}$, methanolwater system] and semi-preparative HPLC [YMC R-ODS-5 AQ, $20 \mathrm{~mm} \times 25 \mathrm{~cm}$, methanol-water system].

Buddlejasaponin IVb (2b) Amorphous powder, $[\alpha]_{\mathrm{D}}^{25}-31.8^{\circ}(c=$ 0.98, methanol). Anal. Calcd for $\mathrm{C}_{48} \mathrm{H}_{78} \mathrm{O}_{18} \cdot 7 / 2 \mathrm{H}_{2} \mathrm{O}: \mathrm{C}, 57.30 ; \mathrm{H}, 8.52$ Found: C, 57.50; H, 8.46. FAB-MS $966[\mathrm{M}+\mathrm{Na}]{ }^{+}$. ${ }^{1} \mathrm{H}-$ and ${ }^{13} \mathrm{C}-\mathrm{NMR}$ : Tables I-III.

Clinoposaponin I (3) Amorphous powder, $[\alpha]_{\mathrm{D}}^{25}+52.4^{\circ} \quad(c=0.41$, methanol). Anal. Calcd for $\mathrm{C}_{48} \mathrm{H}_{78} \mathrm{O}_{18} \cdot 5 \mathrm{H}_{2} \mathrm{O}: \mathrm{C}, 55.80 ; \mathrm{H}, 8.59$. Found: C, 55.85; H, 8.33. FAB-MS: $966[\mathrm{M}+\mathrm{Na}]^{+} .{ }^{1} \mathrm{H}-$ and ${ }^{13} \mathrm{C}-\mathrm{NMR}$ : Tables I-III.

Clinoposaponin II (4) Amorphous powder, $[\alpha]_{\mathrm{D}}^{25}+23.7^{\circ}(c=1.64$, methanol). Anal. Calcd for $\mathrm{C}_{54} \mathrm{H}_{88} \mathrm{O}_{23} \cdot 4 \mathrm{H}_{2} \mathrm{O}$ : C, 55.09; H, 8.22. Found: C, 55.24; H, 8.06. FAB-MS $1128[\mathrm{M}+\mathrm{Na}]^{+} .{ }^{1} \mathrm{H}-$ and ${ }^{13} \mathrm{C}-\mathrm{NMR}$ : Tables I-III.

Clinoposaponin III (5) Amorphous powder, $[\alpha]_{D}^{25}+36.4^{\circ}(c=1.24$, methanol). Anal. Calcd for $\mathrm{C}_{54} \mathrm{H}_{88} \mathrm{O}_{23} \cdot 11 / 2 \mathrm{H}_{2} \mathrm{O}: \mathrm{C}, 53.85 ; \mathrm{H}, 8.29$. Found: C, 53.86; H, 8.22. FAB-MS: $1128[\mathrm{M}+\mathrm{Na}]^{+}$. ${ }^{1} \mathrm{H}-$ and ${ }^{13} \mathrm{C}-\mathrm{NMR}$ : Tables I-III.

Clinoposaponin IIIb (5b) Amorphous powder, $[\alpha]_{\mathrm{D}}^{25}+24.2^{\circ}(c=0.65$, methanol). Anal. Calcd for $\mathrm{C}_{54} \mathrm{H}_{88} \mathrm{O}_{23} \cdot 5 \mathrm{H}_{2} \mathrm{O}: \mathrm{C}, 54.26 ; \mathrm{H}, 8.26$. Found: C, 54.31; H, 8.21. FAB-MS: $1128[\mathrm{M}+\mathrm{Na}]^{+}$. ${ }^{1} \mathrm{H}$ - and ${ }^{13} \mathrm{C}-\mathrm{NMR}$ : Tables I-III.

Clinoposaponin IV (6) Amorphous powder, $[\alpha]_{\mathrm{D}}^{25}+55.7^{\circ}(c=0.70$, methanol). Anal. Caled for $\mathrm{C}_{54} \mathrm{H}_{88} \mathrm{O}_{23} \cdot 6 \mathrm{H}_{2} \mathrm{O}$ : C, 53.45; H, 8.31. Found: C, 53.42; H, 8.10. FAB-MS: $1128[\mathrm{M}+\mathrm{Na}]^{+} .{ }^{1} \mathrm{H}$ - and ${ }^{13} \mathrm{C}-\mathrm{NMR}$ : Tables I-III.

Clinoposaponin V (7) Amorphous powder, $[\alpha]_{\mathrm{D}}^{25}+27.0^{\circ}(c=0.98$, methanol). Anal. Calcd for $\mathrm{C}_{60} \mathrm{H}_{98} \mathrm{O}_{28} \cdot 7 \mathrm{H}_{2} \mathrm{O}: \mathrm{C}, 51.71 ; \mathrm{H}, 8.10$. Found: C, 51.65; H, 7.96. FAB-MS: $1290[\mathrm{M}+\mathrm{Na}]{ }^{+} .{ }^{1} \mathrm{H}-$ and ${ }^{13} \mathrm{C}-\mathrm{NMR}$ : Tables I-III.
Clinoposaponin Va (7a) Amorphous powder, $[\alpha]_{\mathrm{D}}^{25}+38.9^{\circ}(c=0.48$, methanol). Anal. Calcd for $\mathrm{C}_{61} \mathrm{H}_{102} \mathrm{O}_{29} \cdot 7 \mathrm{H}_{2} \mathrm{O}: \mathrm{C}, 51.39 ; \mathrm{H}, 8.20$. Found: C, 51.31; H, 7.92. FAB-MS: $1322[\mathrm{M}+\mathrm{Na}]{ }^{+} .{ }^{1} \mathrm{H}-$ and ${ }^{13} \mathrm{C}-\mathrm{NMR}$ : Tables I-III.

Clinoposaponin Vb (7b) Amorphous powder, $[\alpha]_{\mathrm{D}}^{25}-31.3^{\circ}(c=0.90$, methanol). Anal. Calcd for $\mathrm{C}_{60} \mathrm{H}_{98} \mathrm{O}_{28} \cdot 7 \mathrm{H}_{2} \mathrm{O}: \mathrm{C}, 51.71 ; \mathrm{H}, 8.10$. Found: C, 51.70; H, 8.00. FAB-MS: $1290[\mathrm{M}+\mathrm{Na}]^{+} .{ }^{1} \mathrm{H}$ - and ${ }^{13} \mathrm{C}-\mathrm{NMR}$ : Tables I-III.

Mild Methanolysis and Subsequent Acid Hydrolysis of 1-7 A sample of each compound ( $c a 0.5 \mathrm{mg}$ ) was refluxed with AcCl-methanol $(1: 20)$ $(1 \mathrm{ml})$ for $3 \mathrm{~h}$. The reaction mixture was concentrated to yield a residue, which was partitioned between EtOAc and water. The EtOAc layer was concentrated and subjected to HPLC to reveal a peak due to saikogenin A. The retention time of this material was in agreement with that of authentic saikogenin $\mathrm{A}$. The water layer was concentrated and the residue was heated at $100^{\circ} \mathrm{C}$ with $5 \% \mathrm{H}_{2} \mathrm{SO}_{4}$ aq. ( 2 drops) for $30 \mathrm{~min}$. The reaction mixture was diluted with water and passed through an Amberlite IR-45 column. The eluate was concentrated to give a residue, which was reduced with $\mathrm{NaBH}_{4}(c a . l \mathrm{mg})$ in water $(0.2 \mathrm{ml})$ for $1 \mathrm{~h}$ at room temperature and passed through an Amberlite IR-120 column. The eluate was concentrated to dryness under reduced pressure and then boric acid was removed by co-distillation with methanol. The residue was acetylated with $\mathrm{Ac}_{2} \mathrm{O}$ pyridine (1:1) ( 2 drops) at $100^{\circ} \mathrm{C}$ for $1 \mathrm{~h}$. The reagents were evaporated and the residual alditol acetates were analysed by GC. HPLC conditions: column, YMC Pack R-ODS-7, $4.6 \mathrm{~mm} \times 25 \mathrm{~cm}$; flow rate $1.3 \mathrm{ml} / \mathrm{min}$; solvent, MeCN-water $(55: 45)$. UV $250 \mathrm{~nm} ; t_{\mathrm{R}}$, saikogenin A $14.0 \mathrm{~min}$. GC conditions: column, Supelco SP-2380, $0.25 \mathrm{~mm} \times 30 \mathrm{~m}$; column temperature, $250^{\circ} \mathrm{C}$; carrier gas, $\mathrm{N}_{2} ; t_{\mathrm{R}}$, fucitol acetate $5.1 \mathrm{~min}$, glucitol acetate $10.6 \mathrm{~min}$.

Acknowledgement We are grateful to the staff of the Central Analytical Laboratory of this university for elemental analysis and measurement of MS.

\section{References}

1) A. Yamamoto, T. Miyase, A. Ueno and T. Maeda, Chem. Pharm. Bull., 39, 2764 (1991).

2) H. Ishii, M. Nakamura, S. Seo, K. Tori, T. Tozyo and Y. Yoshimura, Chem. Pharm. Bull., 28, 2367 (1980).

3) K. Shimizu, S. Amagaya and Y. Ogihara, Chem. Pharm. Bull., 33, 3349 (1985)

4) J.-K. Ding, H. Fujino, R. Kasai, N. Fujimoto, O. Tanaka, J. Zhou, H. Matsuura and T. Fuwa, Chem. Pharm. Bull., 34, 1158 (1986).

5) S.-R. Xue, J.-Q. Liu and G. Wang, Phytochemistry, 31, 1049 (1992). 